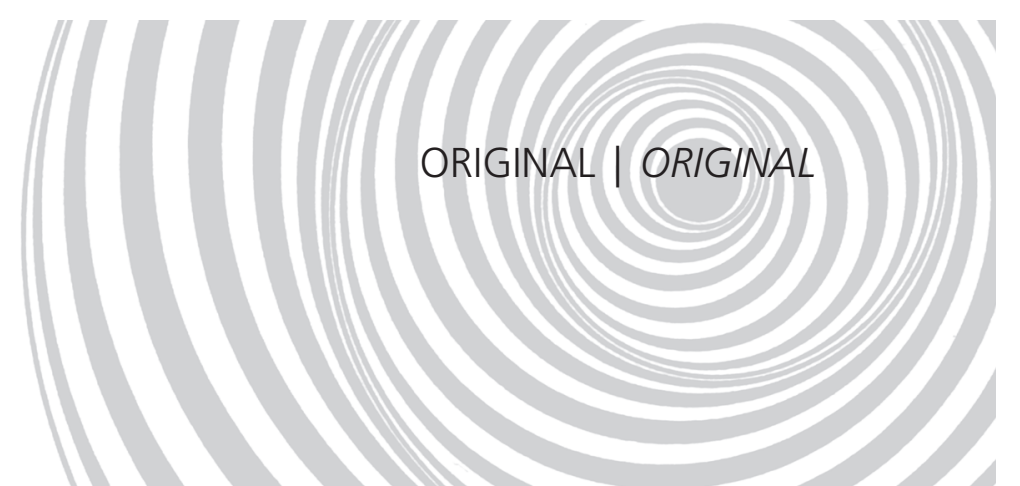

\title{
Avaliação da eficácia e do grau de adesão ao uso prolongado de fibra dietética no tratamento da constipação intestinal funcional ${ }^{1}$
}

\author{
Evaluation of the efficacy and adherence to \\ long-term use of dietary fiber in the treatment \\ of functional intestinal constipation
}

Wellington Monteiro MACHADO2

Sílvia Maria CAPELARI ${ }^{3}$

RE S U M O

\section{Objetivo}

Avaliar o grau de adesão e a eficácia da fibra dietética no tratamento da constipação intestinal funcional.

\section{Métodos}

Estudo clínico longitudinal, envolvendo 59 indivíduos, 54 mulheres e 5 homens, idade média de 46 anos (variação: 18-74 anos), com diagnóstico de constipação intestinal funcional. Após regularização do hábito intestinal, com a introdução de fibra dietética, era recomendado aos pacientes manter a dieta com fibra e retornar quatro meses depois, a fim de ser verificada a continuidade da eficácia e o grau de adesão ao seu uso. Associação entre adesão e eficácia foi pesquisada pelo teste exato de Fisher.

\section{Resultados}

Nove pacientes abandonaram o estudo e 50 continuaram. Destes, 32 mantiveram hábito intestinal satisfatório e a constipação recorreu em 18. Naqueles com manutenção do hábito intestinal, dezesseis mantiveram a ingestão de fibra recomendada, quatro aumentaram, nove reduziram e três interromperam. Nos casos com recorrência do sintoma, a quantidade de fibra foi mantida em três, aumentada em um, diminuída em três e suspensa em onze. Foi encontrada associação estatisticamente significante entre adesão e eficácia $(p=0,008)$.

\footnotetext{
1 Artigo elaborado a partir da dissertação de S.M. CAPELARI, intitulada "Estudo do efeito da fibra dietética conforme o tipo de sintoma da constipação". Universidade Metodista de Piracicaba; 2007.

2 Universidade Estadual Paulista Júlio de Mesquita Filho, Faculdade de Medicina de Botucatu, Departamento de Clínica Médica. Rubião Jr, s/n., 18618-000, Botucatu, SP, Brasil. Correspondência para/Correspondence to: W.M. MACHADO. E-mail: <wmachado@fmb.unesp.br>.

${ }^{3}$ Universidade Metodista de Piracicaba, Curso de Nutrição. Piracicaba, SP, Brasil.
} 


\section{Conclusão}

A eficácia da fibra dietética mostrou-se moderada, ocorrendo benefício em $64 \%$ dos casos. A perda de adesão foi grande, mais da metade dos indivíduos avaliados (52\%). Tais resultados sugerem a necessidade de novas estratégias, visando garantir maior fidelidade à terapêutica com fibra e com isto melhorar seus resultados no tratamento da constipação intestinal.

Termos de indexação: Constipação intestinal. Fibras na dieta. Eficácia da dieta com fibra. Hábitos alimentares.

\section{A B S T R A C T}

\section{Objective}

The objective of this study was to evaluate the adherence to and efficacy of dietary fiber in the treatment of functional constipation.

\section{Methods}

This is a longitudinal clinical study involving 59 subjects, 54 women and 5 men, aged 18 to 74 years, mean age of 46 years, with functional constipation. Once the bowel movements became regular with the use of dietary fiber, subjects were instructed to maintain the fiber-rich diet for four months in order to determine if the efficacy continued over time and the degree of adherence to its use. The association between adherence and efficacy was investigated by the Fisher's exact test.

\section{Results}

Although nine individuals abandoned the study, 50 returned. From these, 32 sustained normal bowel movements and 18 had recurrence of the constipation. In the first group, 16 continued consuming the same amount of fiber, four increased, nine reduced and three stopped taking it. In the group with recurrence, three maintained the same amount of fiber, one increased, three reduced and eleven stopped taking it. There was a significant statistical association between adherence and efficacy $(p=0.008)$.

\section{Conclusion}

The long-term efficacy of dietary fiber for the treatment of constipation was moderate (64\%) and treatment drop-out rate was high (52\%). These results suggest the need of new strategies that promote greater fidelity to fiber therapy, thereby improving the efficacy of constipation treatment.

Indexing terms: Dietary fiber. Constipation. Dietary fiber efficacy. Food habits.

\section{N T R O D U ÇÃ O}

Constipação intestinal é uma condição frequente, acometendo elevado número de indivíduos, num percentual que varia, de acordo com os locais e tipos de inquéritos, entre $2 \%$ a $27 \%$ da população ${ }^{1,2}$. Na maioria das vezes, sua etiologia não é clara, recebendo a classificação de constipação funcional (CF). Em outras, contudo, existe uma causa subjacente, orgânica, à qual é secundária. Embora usualmente não represente risco à vida, a constipação funcional pode provocar grande desconforto nos portadores e repercutir negativamente na sua qualidade de vida e no seu desempenho pessoal ${ }^{3,4}$. O tratamento é comumente baseado em dieta rica em Fibra Dietética (FD), podendo ser suplementado pelo uso de substâncias laxativas ${ }^{5-7}$. As fibras mais frequentemente recomendadas são as insolúveis, cujo sucesso parece estar na dependência direta do uso ininterrupto de cotas diárias elevadas ${ }^{8-10}$.

A obtenção da adesão dos constipados ao uso continuado de fibra não é, contudo, de fácil consecução. Os motivos são variados, indo desde o sabor insípido de produtos como farelo de trigo, até a ocorrência de efeitos desconfortáveis como distensão abdominal, plenitude e flatulência ${ }^{7-10}$. Além disso, há a concorrência vantajosa dos laxantes e supositórios, produtos fáceis de ingerir ou aplicar, de baixo custo e cujos efeitos são imediatos.

A eficácia do uso de fibra dietética na prevenção de doenças crônicas, particularmente 
cardiovasculares ${ }^{11,12}$, pólipos e câncer do cólon ${ }^{13-16}$ tem sido estudada extensivamente. Um dos itens considerados importantes no êxito do tratamento é representado pelo grau de adesão à prescrição. Para consegui-lo, numerosas estratégias têm sido propostas, visando estimular ou garantir a manutenção da fidelidade ao uso continuado da $\operatorname{dieta}^{17-20}$. As estratégias preveem entrevistas prolongadas com os indivíduos estudados, treinamento no preparo dos alimentos apropriados, cardápios bem definidos, contatos pessoais frequentes da equipe responsável com os indivíduos envolvidos, visando ao reforço dos compromissos assumidos, intervenções comportamentais e, por fim, dosagem de biomarcadores no sangue ou na urina, capazes de confirmar o consumo regular dos alimentos prescritos ${ }^{11,21-23}$.

Surpreendentemente, embora utilizada em larga escala no tratamento da CF, a avaliação da eficácia prolongada da dieta rica em fibra não tem merecido muitos estudos. Igualmente, têm sido escassas as investigações a respeito do grau de adesão dos usuários a essa modalidade terapêutica. Ambas, eficácia duradoura e grau de adesão são, no entanto, parâmetros importantes no julgamento do efeito das fibras na constipação e, portanto, necessitam ser conhecidos com maior precisão. Só o domínio aprofundado e consistente do tema permitirá conclusões sobre a real efetividade e os tipos de dificuldades a serem enfrentadas pelos praticantes dessa opção terapêutica.

Este trabalho tem por fim averiguar a estabilidade da eficácia de uma dieta rica em fibra dietética no tratamento da constipação funcional e caracterizar o grau de adesão dos usuários a esse tipo de abordagem.

\section{MÉ T O D O S}

Para este estudo clínico longitudinal em dois momentos, foram recrutados 59 indivíduos adultos, com diagnóstico de constipação funcional, definida de acordo com as recomendações estabelecidas pelo Consenso de Roma $11^{24}$, relativas às desordens funcionais digestivas. $\mathrm{O}$ grupo era constituído de 54 mulheres e 5 homens, com idade média de 46 anos (variação de 18-74 anos). Sob a orientação de uma nutricionista, foram todos incluídos num programa de uso de quantidades crescentes de fibra, predominantemente derivada do farelo de trigo. A fibra dietética era ofertada em doses diárias, aumentadas $4 \mathrm{~g} / \mathrm{d}$ quinzenalmente, até atingir um valor julgado suficiente pelo paciente e pela nutricionista assistente na restauração de um Hábito Intestinal (HI) satisfatório. Embora parcialmente subjetiva, a noção de hábito intestinal satisfatório foi escolhida como parâmetro de desfecho do tratamento, por ser capaz de traduzir a recuperação do bem estar intestinal de cada indivíduo, independente de ele se basear em frequência evacuatória ou nas outras variadas sensações desencadeadas pela defecação, tais como evacuação incompleta, esforço exagerado de expulsão das fezes, ressecamento do material fecal ${ }^{24}$. Quando atingido o grau de satisfação, no qual o paciente afirmava que o sintoma que o importunava havia cessado, a situação era considerada como êxito terapêutico. Nesse momento, após minuciosa e esclarecedora entrevista, eram instruídos a não abandonar a dieta, mantendo-a nos moldes que assegurara seu sucesso até então, os pacientes recebiam alta temporária do ambulatório, com consulta agendada para quatro meses depois, encerrando assim uma primeira fase de abordagem. Ao retornarem no prazo estipulado, cumpriam a segunda fase da abordagem, na qual eram submetidos a nova e cuidadosa entrevista, onde, baseada em um questionário semiestruturado (Anexo), era avaliada a permanência da eficácia da dieta e verificado o grau de adesão ao seu uso.

Para efeito deste trabalho, foi definido como eficácia a manutenção de um HI satisfatório após quatro meses contínuos de tratamento. Foi julgado como perda de adesão a redução ou suspensão da quantidade diária de fibra dietética, definida ao fim da primeira fase de tratamento como a dose apropriada para a normalização do Hábito Intestinal.

Foi investigado a existência de associação entre adesão e eficácia por intermédio do teste 
exato de Fisher e calculada a razão de risco referente à eficácia em função da perda ou manutenção da adesão à dieta. $\mathrm{O}$ nível de significância estatística aceito foi de $p<0,05$.

Este trabalho foi aprovado pelo Comitê de Ética em Pesquisa do Hospital das Clínicas de Botucatu (protocolo $n^{\circ}$ 1099) sendo obrigatório, para dele participar, a assinatura de um termo de consentimento livre e esclarecido.

\section{RES ULTADOS}

Dos 59 indivíduos incluídos, nove não regressaram ao fim dos quatro meses. Esse grupo era constituído de um homem e oito mulheres, com idade média de 50,5 anos (variação de 34-71 anos), distinguindo-se dos que permaneceram em acompanhamento, pela média de idade mais alta e menor proporção de mulheres.

Dos 50 que retornaram, 4 eram homens e 46 mulheres, idade média de 45,8 anos (variação de 18-74 anos). Desse total, 32 mantiveram hábito intestinal satisfatório e 18 apresentaram recorrência da constipação. Dos que conservaram o hábito intestinal normal, 16 mantiveram o consumo de fibra na quantidade recomendada, quatro aumentaram, nove reduziram e três abandonaram seu uso. Naqueles com recorrência do sintoma, o consumo de fibra foi mantido na dose indicada em três, aumentou em um, diminuiu em três e foi interrompido em 11 (Tabela 1). A partir desses dados e adotada a análise por protocolo, o índice de eficácia obtido foi de $64 \%$ e o de perda de adesão de $52 \%$. A associação entre essas duas variáveis foi estatisticamente significante $(p=0,008)$ e a razão de risco, referente à ocorrência de hábi- to intestinal insatisfatório, foi 3 vezes maior para o grupo que não aderiu ao uso de fibra dietética (Tabela 2).

Na entrevista de retorno, os pacientes em que foi constatada inobservância ao esquema dietético preconizado foram questionados sobre as razões desse comportamento, sendo apresentadas como justificativas: desprazer com a ingestão da dieta, piora ou surgimento de sintomas abdominais associados ao consumo da fibra dietética, indiferença às orientações traçadas pelo profissional assistente e quadros de ansiedade originados pela ocorrência de irregularidade do hábito intestinal.

\section{I S CUSS Ã O}

Dos indivíduos recrutados para o estudo, 15,2\% o abandonaram. Possuíam características demográficas discretamente diferentes daqueles que permaneceram, impossibilitando que conclusões aplicadas a esses últimos pudessem a eles ser estendidas.

Analisando o grupo que permaneceu no estudo e julgando o grau de eficácia terapêutica da fibra dietética, por um período de tempo de média duração, os resultados mostraram saldo moderadamente favorável, uma vez que somente 32 dos 50 indivíduos acompanhados preservaram o hábito intestinal ao longo do período de observação. A maior taxa de sucesso ocorreu entre os que mantiveram o consumo de fibra dietética, o que está de acordo com o achado de associação significante $(p=0,008)$ entre adesão e eficácia da fibra dietética. Em alguns casos, no entanto, apesar da redução ou suspensão da fibra dietética,

Tabela 1. Dados demográficos e consumo de fibra dietética, após quatro meses de tratamento, de indivíduos com recorrência ou controle adequado da constipação. Botucatu (SP), 2007.

\begin{tabular}{|c|c|c|c|c|c|c|c|c|c|c|c|c|c|}
\hline \multirow{3}{*}{ Constipação } & \multirow[b]{3}{*}{$\mathrm{n}$} & \multirow[b]{3}{*}{$\%$} & \multirow{3}{*}{$\begin{array}{c}\text { Idade média/ano } \\
\text { (faixa etária) }\end{array}$} & \multirow{2}{*}{\multicolumn{2}{|c|}{ Sexo }} & \multicolumn{8}{|c|}{ Consumo de fibra (\%) } \\
\hline & & & & & & \multicolumn{2}{|c|}{ Aumentou } & \multicolumn{2}{|c|}{ Diminuiu } & \multicolumn{2}{|c|}{ Manteve } & \multicolumn{2}{|c|}{ Suspendeu } \\
\hline & & & & M & $\mathrm{F}$ & $\mathrm{n}$ & $\%$ & $\mathrm{n}$ & $\%$ & $\mathrm{n}$ & $\%$ & $\mathrm{n}$ & $\%$ \\
\hline Recorrente & 18 & 36 & $44,6(18-70)$ & 1 & 17 & 1 & 5,6 & 3 & 16,7 & 3 & 16,7 & 11 & 61,1 \\
\hline Controlada & 32 & 64 & $32,6(18-74)$ & 3 & 29 & 4 & 12,5 & 9 & 28,1 & 16 & 50,0 & 3 & 9,4 \\
\hline
\end{tabular}


Tabela 2. Associação entre eficácia e adesão ao uso de Fibra Dietética no tratamento da constipação. Botucatu (SP), 2007.

\begin{tabular}{|c|c|c|c|c|c|c|}
\hline \multirow{3}{*}{ Adesão } & \multicolumn{4}{|c|}{ Eficácia } & \multirow{2}{*}{\multicolumn{2}{|c|}{ Total }} \\
\hline & \multicolumn{2}{|c|}{ Não } & \multicolumn{2}{|c|}{ Sim } & & \\
\hline & $\mathrm{n}$ & $\%$ & $n$ & $\%$ & $n$ & $\%$ \\
\hline Não & 14 & 53,8 & 12 & 46,2 & 26 & 100,0 \\
\hline Sim & 4 & 16,6 & 20 & 83,3 & 24 & 100,0 \\
\hline Total & 18 & 36,0 & 32 & 64,0 & 50 & 100,0 \\
\hline
\end{tabular}

Teste exato de Fisher: $p=0,008$

foi observado um bom desempenho intestinal. É provável que tal desfecho se deva, em parte, à mudança de estilo de vida e adoção de uma dieta melhor balanceada, implementados espontaneamente pelos pacientes, influenciados pelo trabalho de orientação educacional e nutricional recebidos durante a fase inicial do estudo.

Nos 18 pacientes, nos quais a eficácia da fibra dietética se mostrou insuficiente, a razão principal foi a interrupção completa ou parcial do seu consumo, traduzindo, provavelmente, mais uma perda de adesão do que propriamente ineficácia da terapia prescrita. A razão de risco, aplicada à casuística, demonstrou que a falta de adesão associa-se à acentuada queda da eficácia. Em alguns dos pacientes, contudo, apesar do rigoroso cumprimento das doses recomendadas e, até mesmo, do aumento da quantidade ingerida, houve recorrência da constipação, tornando patente a inefetividade das fibras em alguns casos.

Com relação ao aspecto adesão, um fato importante a merecer comentário foi a taxa de abandono do acompanhamento após a primeira fase. Considerar, no entanto, o abandono como uma forma de perda de adesão é questionável, já que são desconhecidos os motivos que levaram os indivíduos a desistirem do estudo. Razões podem, no entanto, ser presumidas, tais como o desaparecimento do sintoma, induzindo ao sentimento de desnecessidade de novas consultas. Outra hipótese a ser cogitada é a da prática da automedicação, hábito bem difundido entre nós e com muitos adeptos, sobretudo quando se trata de afecção corriqueira e de baixo risco, como a constipação intestinal. Embora razoáveis, essas, bem como outras suposições, necessitariam de confirmação para terem validade, o que é inexequível no presente contexto.

Do total de 50 indivíduos estudados, a maioria perdeu a adesão, seja por redução ou abandono completo da dieta prescrita. Entre as variadas justificativas referidas, a mais relevante foi a intolerância ao uso continuado da fibra dietética, o que não é surpresa, se levado em conta a monotonia do uso diário do farelo de trigo, seu aspecto e sabor pouco atrativos. Adicionalmente, efeitos característicos das fibras, como flatulência e distensão, podem contribuir para reforçar a oposição ao seu uso sistemático ${ }^{7-9}$. Outro motivo alegado foi a resistência-negligência ao cumprimento de regras ou normas estabelecidas. Esse é um comportamento não raro entre alguns pacientes e do conhecimento de qualquer profissional de saúde ${ }^{25,26}$, não sendo, portanto, surpreendente que ocorra com a prescrição de rotinas dietéticas. Por fim, quadros de ansiedade também foram apontados como motivo de ruptura da adesão. A ansiedade pode ocorrer em razão do próprio perfil emocional descrito em pacientes constipados ${ }^{27}$, assim como pela percepção alterada apresentada por parte deles diante do quadro de constipação, em que ela é vista como potencial fonte de produtos tóxicos, derivados das fezes retidas no cólon por tempo prolongado ${ }^{28}$. Para tais indivíduos, a constipação interfere no seu bem estar geral e deve ser corrigida preferencialmente por medidas de efeito imediato, tipo drogas laxativas, e não por intermédio de produtos de ação gradual como alimentos ricos em fibra dietética $6,7,27,28$.

Dos 26 indivíduos com adesão comprometida, 14 apresentaram recorrência da constipação, enquanto os demais mantiveram o hábito intestinal normal. Essa última situação é curiosa, e explicações para ela são semelhantes às já descritas anteriormente em relação à eficácia, em que, apesar da redução ou supressão do consumo de fibra dietética, alguns pacientes conservaram normal o funcionamento intestinal. 
Um outro ponto relevante refere-se à escolha do intervalo de quatro meses como prazo adequado para avaliação da adesão e eficácia da fibra dietética. A opção deste estudo, foi determinada com o fim de garantir um período de análise suficientemente longo, no caso 16 semanas, mas não tão extenso que favorecesse a evasão dos indivíduos sob observação. É bem conhecida a tendência dos pacientes em desconsiderar progressivamente o cumprimento das prescrições médicas à medida que se alarga o intervalo das consultas, assim como a dificuldade de alguns em frequentar serviços de saúde com assiduidade. O período de quatro meses pareceu adequado, neste estudo para garantir não só maior fidelidade aos retornos, como também um monitoramento adequado da evolução do tratamento. Ademais, a literatura internacional tem utilizado grande variedade de duração de intervalos no acompanhamento de programas relativos à adesão e à eficácia de dietas. Assim, enquanto alguns protocolos requerem anos para atingir seus objetivos, como os dedicados ao estudo da prevenção de pólipos intestinais ${ }^{13,15}$, em outros intervalos menores são utilizados, medidos em meses $^{29}$ e mesmo em semanas de duração ${ }^{30}$.

\section{O NCLUSÃ O}

A dieta baseada em fibra dietética mostrou moderado grau de eficácia no tratamento da constipação funcional, quando aplicada por período de tempo prolongado. A baixa adesão, observada em mais da metade dos pacientes, foi um importante fator contribuinte para esse desfecho.

Considerando a elevada prevalência da constipação funcional e a necessidade de boas opções terapêuticas, justifica-se um redobrado esforço para melhorar os índices de adesão e eficácia da fibra dietética. Para tanto, novas estratégias devem ser desenvolvidas, visando garantir maior fidelidade ao consumo prolongado da dieta com fibra.

\section{COLABORADORES}

W.M. MACHADO concebeu o projeto, o planejamento, a análise dos dados e a redação do texto final. S.M. CAPELARI concebeu o delineamento metodológico, a coleta e a análise dos dados e a redação do texto final.

\section{REFERÊ NCIAS}

1. Garrigues V, Galvez C, Ortiz V, Ponce M, Nos P, Ponce J. Prevalence of constipation: agreement among several criteria and evaluation of the diagnostic accuracy of qualifying symptoms and self-reported definition in a population-based survey in Spain. Am J Epidemiol. 2004; 159(5): 520-6.

2. Pare P, Ferrazzi S, Thompson WG, Irvine EJ, Rance L. An epidemiological survey of constipation in Canada: definitions, rates, demographics, and predictors of health care seeking. Am J Gastroenterol. 2001; 96(11):3130-7.

3. Glia A, Lindberg G. Quality of life in patients with different types of functional constipation. Scand J Gastroenterol. 1997; 32(11):1083-9.

4. Wald A, Scarpignato C, Kamm MA, Mueller-Lissner A, Helfrich I, Schwijt C, et al. The burden of constipation on quality of life: results of a multinational survey. Aliment Pharmacol Ther. 2007; 26(2):227-36 doi: 10.1111/j.1365-2036. 2007.03376.x.

5. Locke III GR, Pemberton JH, Phillips SF. AGA technical review on constipation. Gastroenterology. 2000; 119(6):1766-78.

6. Ramkumar D, Rao SSC. Efficacy and safety of traditional medical therapies for chronic constipation: a systematic review. Am J Gastroenterol. 2005; 100(4):936-71. doi:10.1111/j.1572-0241.2005.40 925.

7. Schiller LR. Review article: the therapy of constipation. Aliment Pharmacol Ther. 2001; 15(6):749-63. doi: 10.1046/j.1365-2036.2001.0 0982.x.

8. Voderholzer WA, Schatke W, Muhldorfer BE, Klauser AG, Birkner B, Muller-Lissner SA. Clinical response to dietary fiber treatment of chronic constipation. Am J Gastroenterol. 1997; 92(1): 95-8.

9. Kritchevsky D. Dietary fiber. Ann Rev Nutr. 1988; 8:301-28.

10. Jenkins DJA, Peterson RD, Thorne MJ, Ferguson PW. Wheat fiber and laxation: dose response and 
equilibrium time. Am J Gastroenterol. 1987; 82(12): 1259-63.

11. Gleason JA, Bourdet KL, Koehn K, Holay S, Schafer EJ. Cardiovascular risk reduction and dietary compliance with a home-delivered diet and lifestyle modification program. J Am Diet Assoc. 2002; 102(10):1445-51

12. Solá R, Godás G, Ribalta J, Valvé JC, Girona J, Anguera $A$, et al. Effects of soluble fiber (Plantago ovata husk) on plasma lipids, lipoproteins, and apolipoproteins in men with ischemic heart disease. Am J Clin Nutr. 2007; 85(4):1157-63.

13. Alberts DS, Martinez ME, Roe DJ, Guillen-Rodriguez $J M$, Marshall JR, van Leeuwen JB, et al. Lack of effect of a high-fiber cereal supplement on the recurrence of colorectal adenomas. N Engl J Med. 2000; 342(16):1156-62.

14. Lanza E, Schatzkin A, Daston C, Corle D, Freedman $\mathrm{L}$, Ballard-Barbash $\mathrm{R}$, et al. Implementation of a 4-y, high-fiber, high-fruit-and-vegetable, low-fat dietary intervention: results of dietary changes in the Polyp Prevention Trial. Am J Clin Nutr. 2001; 74(3):387-401.

15. Schatzkin A, Lanza E, Corle D, Lance P, Iber F. Lack of effect of a low-fat, high-fiber diet on the recurrence of colorectal adenomas. $\mathrm{N}$ Engl J Med. 2000; 342(16):1149-55.

16. Larsson SC, Giovanucci E, Bergkvist L, Wolk A. Whole grain consumption and risk of colorectal cancer: a population-based cohort of 60,000 women. Br J Cancer. 2005; 92(9):1803-7. doi: 10.1038/sj.bjc.6602543.

17. Brownell KD, Cohen LR. Adherence to dietary regimens 1: an overview of research. Behav Med. 1995; 20(4):149-54.

18. Glanz K. Compliance with dietary regimens: Its magnitude, measurement, and determinants. Prev Med. 1980; 9(6):787-804.

19. Jeffery RW, Bjornson-Benson WM, Rosenthal BS, Lindquist RA, Kurth $\mathrm{CL}$, Johnson SL. Correlates of weight loss and its maintenance over two years of follow-up among middle-aged men. Prev Med. 1984; 13(2):155-68.

20. Brownell KD, Cohen LR. Adherence to dietary regimens 2: components of effective interventions. Behav Med. 1995; 20(4):155-64.

21. Bernstein MA, Nelson ME, Tucker KL, Layne J, Johnson E, Nuernberger A, et al. A home-based nutrition intervention to increase consumption of fruits, vegetables, and calcium-rich foods in community dwelling elders. J Am Diet Assoc. 2002; 102(10):1421-7.

22. Ammerman AS, Lindquist CH, Lohr KN, Hersey J. The efficacy of behavioral interventions to modify dietary fat and fruit and vegetable intake: a review of the evidence. Prev Med. 2002; 35(1):25-41. doi:10.1006/pmed.2002.1028.

23. Lanza E, Schatzkin A, Ballard-Barbash R, Clifford DC, Paskett E. The polyp prevention trial II: dietary intervention program and participant baseline dietary characteristics. Cancer Epidemiol, Biomakers and Prev. 1996; 5(5):385-92.

24. Thompson WG, Longstreth GF, Drossman DA, Heaton KW, Irvine EJ, Mueller-Lissner SA. Functional bowel disorders and functional abdominal pain. Gut. 1999; 45(2):1143-7.

25. Kane S. Just a spoonful of sugar helps the medicine go down. If only it was that simple! nonadherence in inflmmatory bowel disease. Am J Gastroenterol. 2007; 102(7):1427-8. doi: 10.1111/j.15720241.20 07.01213.x.

26. Graham DY, Lew GM, Malaty HM, Evans DG, Evans DJ Jr, Klein PD, et al. Factors influencing the eradication of Helicobacter pylori with triple therapy. Gastroenterology. 1992; 102(2):493-6.

27. Mason HJ, Serrano-Ikkos E, Kamm MA. Psychological morbidity in women with idiopathic constipation. Am J Gastroenterol. 2000; 95(10): 2852-7. doi:10.1111/j.1572-0241.2000.02313.x.

28. Muller-Lissner SA, Kamm MA, Scarpignato C, Wald A. Myths and misconceptions about chronic constipation. Am J Gastroenterol. 2005; 100(1): 232-42. doi:10.1111/j.1572-0241.2005.40885.x.

29. Richards A, Kattelmann KK, Ren C. Motivating 18- to 24-year-olds to increase their fruit and vegetable consumption. J Am Diet Assoc. 2006; 106(9):1405-11. doi:10.1016/j.jada.2006.06.005.

30. Chan AO, Leung G, Tong T, Wong NYH. Increasing dietary fiber intake in terms of kiwifruit improves constipation in Chinese patients. World J Gastroenterol. 2007; 13(35):4771-5.

Recebido em: 20/4/2008

Versão final reapresentada em: 25/11/2008 Aprovado em: 16/6/2009 
238 | W.M. MACHADO \& S.M. CAPELARI

ANEXO

PROTOCOLO DE RETORNO DO TRATAMENTO DA CONSTIPAÇÃO

Nome: RG:

Data:

* Fonte e quantidade de fibra que utilizou:

A) Adequação à dieta anterior: ( ) respondeu ( ) suspendeu ( ) não respondeu

B) Resposta às medidas terapêuticas: ( ) melhorou ( ) piorou ( ) inalterado

C) Por que melhorou?

D) Por que piorou?

E) Uso de laxantes:
( ) aumentou
( ) diminuiu
( ) inalterado
( ) não usou

F) Quantidade aproximada de líquido consumido diariamente?

G) A observância do paciente às medidas recomendadas foi:
( ) satisfatória
( ) irregular
( ) ignorou

H) Comentários e conclusões sobre a consulta e o próximo passo a ser dado como conduta 